2.

Erwiderung anf Herrn Prof. Fr. Merkel's Bemerkungen zu meiner Abhandlung: „Die Gestalt des oberen Theils der Hinterhanptsschnppe als Charakteristikon des menschlichen Koples."

(Cf. Dieses Archiv Bd. LIX. Hft. 2. S. 297. 1874.)

Von Dr. G. Joseph,

pract. Arzte und Docenten an der Universität zu Breslau.

Die Bemerkungen, welche Herr Prof. Fr. Merkel zu Seite 6-11 der bezeichneten Abliandlung gemacht hat, weise ich in Nacbstehendem, als unzutreffend, zurück.

Zunächst behauptet derselbe, dass ich zu den in seiner Brochüre angegebenen Thatsachen "nichts Neues" zuzufügen hatte und nur gesagt habe, dass die in seinem Schriftchen mitgetheilten Raceneigenthümlichkeiten nicht in der behaupteten Ausdelunung (und Abgrenzung) vorhanden seien. Abgesehen davon, dass einschränkende oder negirende Befunde (welche zuweilen ebenso wichtig sind, als bestätigende) als etwas in der Brocbüre des Herrn Prof. M. nicht Vorhandenes, also doch als etwas Neues, angesehen werden müssen, labe ich auch neue Thatsachen mitgetheilt. Bekanntlich bemerkt man an der Hinterhauptsschuppe, besonders bei männlichen Schädeln niedrigster Menschenraçen, zuweilen eine 0,5 bis 1,5 Centimeter und darüber breite Knochenleiste, durch welche die Linea semicircularis superior nach oben verbreitert erscheint. Ich habe nun wabrgenommen ${ }^{\mathbf{1}}$ ), dass diese Knocbenleiste an Schädeln von Malayen häufig durch eine Furche in eine obere und eine untere getrennt ist. Die untere ist die Linea semicircularis superior; die obere ist die an Schädeln von Europäern an Umfang reducirte Linea semicircularis suprema etc. Dieser am ausgeprägtesten an Malayenschädeln wabrgenommene Befund findet sich in des Herrn Prof. M. Schrift nicht erwähnt. Ferner habe ich mitgetheilt, dass die Protuberantia occip. ext., welche Herr Prof. M. der Mitte der Linea semicirc. suprema zuweist, häufiger der Mitte der Linea semicirc. superior, selten keiner der beiden Linien angehört und in letzterem Falle so gelegen ist, dass die eine darüber, die andere darunter hinwegläuft etc.

Sodann soll ich nach Herrn Prof. M. versäumt haben, statistische Angaben ïber das verwendete anthropologische Material zu machen. Diese Behauptung wird z. B. durch Seite 7 meiner Arbeit widerlegt: „Andeutungen der leistenförmigen Knochenauftreibung zeigten sich unter 642 (europ.) Schädeln $16 \mathrm{mal}$ (2,5 pCt.) und der mit der Mittelfurche versehenen $24 \mathrm{mal}(3,73 \mathrm{pCt}$.) " etc. Für meine Arbeit, welche die Darstellung der Unterschiede in der Entwickelung der Hinterhauptsschuppe des Menschen und der Affen von einer bestimmten Lebenszeit ab bezweckte, war, im Gegensatze zu dem Zwecke der Arbeit des Herrn Prof. Merkel, der Nachweis der Existenz einer dritten transversalen Knochenlinie an deren Oberdăche ein nur untergeordnetes, nebensächliches Moment. Für meinen Zweck

$\left.{ }^{1}\right)$ Cf. S. 7 meiner Schrift: Morpholog. Studien am Kopfskelet des Menschen und der Wirbelthiere. Breslau. Korn. 1873. 
genügten kurze statistische Notizen darüber. Die Besprechung der letztgenannten Linie von Seite 6 bis 11 in meiner Arbeit gegenüber den 22 Seiten, welche dieselbe im Ganzen umfasst, deutet darauf ebenfalls hin und dürfte den Leser über die Richtigkeit des Urtheils des Herrn Prof. M., dass das Ergebniss meiner Wahrnehmungen ein mageres sei, aufklären. Ueberhaupt rührt die Veranlassung zu seinen unbegründeten Ausstellungen an meiner Arbeit von gänzlichem Verkennen der Verschiedenheit unserer Tendenzen her. Für Herrn Prof. M. war die Veröffentlichung der neuen Linie und der Nachweis ihrer eminenten anatomischen und anthropologischen Wichtigkeit alleiniger Zweck seiner Arbeit. Das lag mir aber ganz fern. Ganz im Gegentheile dazu war ich bestrebt nachzuweisen, 1) dass an der Hinterbauptsschuppe sich Nichts finde, was für sich allein als absolute Raceneigenthümlichkeit betrachtet werden könne (cf. S. 12 meiner Schrift), 2) dass aber nach der Geburt der Kopf des Menschen und derjenige der Affen entgegengesetzte Wege des Wachsthums gehen, dass in letzterem charakteristische Unterschiede auftreten, weIche die Gestaltung der in Rede stebenden Knochenplatte beim Menschen von der bei den Affen streng scheiden. Während Herr Prof. M. in den verschiedenen Ausbildungsgraden der neuen Linie (und des Höckers in ihrer Mitte) 1) gut umgrenzte Raceneigenthümlichkeiten und 2) in dem Occipital-Kamm der Affen, sowie in dem in der Mitte desselben befindlichen Höckerchen (Tuberculum linearum nach M.) den Uebergang zu der Bildung bei niederen Menschenracen erblickt, ist meine Anschauung davon eine andere. Ich sehe 1) die Variationen der Linea semicirc. suprema (so wie die der superior) bei verschiedenen Rácen durch Uebergänge deutlich überbrückt und erkenne in denselben - man entschuldige diese Wiederholung - für sich allein keine anthropologische Marke von unzweifelhaftem Werthe. Die gegentheiligen Schlussfolgerungen des Herrn Prof. M. zähle ich zu den übereilten Dogmen, an denen die Antbropologie mehr als reich ist. 2) In dem allmăhlichen Verschwinden bis zum fast spurlosen Untergange sowohl der angedeuteten L. semicirc. suprema, als der deutlich ausgebildeten superior in der Crista lambdoidea der Affen im Gegensatze $z$ u dem lebenslänglichen Bestehen dieser Linien etc. beim Menschen erkenne ich einen der durcbgreifenden Unterschiede in dem Entwickelungsgange beider Wesenreihen nach der Geburt, der durch keine Uebergänge vermindert wird. Das Höckerchen in der Mitte des OccipitalKammes als Erinnerung an die ehemals von letzterem getrennte Protuberantia occipitalis externa und noch einige andere, wle es scheint, Herrn Prof. M. unbekannte, Erscheinungen bleiben eben als letzte Reste ebemals nabestehender Anfänge, als Zeichen divergent liegender Ziele der Ausbildung nach der Geburt bestehen. Jenes Höckerchen, nach meiner Auffassung die ehemalige Protuberanz, von Herrn Prof. M. Tuberculum linearum genannt [von welchem letzterer irrthümlich (cf. S. 20 meiner Schrift) behauptet, dass es mir entgangen sei], welches an der menschlichen Hinterhauptsschuppe mit zunehmendem Alter bis zum vollendeten Wachsthum durch eine immer grössere Distanz von der Mitte der Lambdanaht getrennt erscheint, rückt dagegen bei der Hinterhauptsschuppe, am auffallendsten bei den anthropomorphen Affenarten, dieser Mitte immer näher, um endlich - in einer dem Menschenbaften völlig fremden Entwickelung - mit dieser Mitte zu verschmelzen (cf. S. 19-22) meiner Schrift). Die meiste Annäherung in diesem Punkte, sowie in 
der Gestaltung der Hinterhauptsschuppe (überhaupt des ganzen Kopfskelets), erscheint in der Jugend der Wesen. Je älter dieselben werden, desto grössere morphologische Divergenz, wodurch bis auf allmählich geringere Spuren, ja bis zur Unkenntlicbkeit, die Zeichen einstiger menschenbaften Anfänge verwischt werden. Die Darstellung der Gesetze, nach welchen diese Phänomene sich vollziehen, und welche der Tendenz des M.'schen Schriftchens so fern, als möglich, liegt, bildet das Hauptthema meiner Arbeit. Mögen dieselben in der ursprünglichen Form, an der ich jetzt Manches zu verbessern hätte, aus meinem am 8 . März 1872 in der schlesischen Gesellschaft für vaterländische Cultur gehaltenen Vortrage wörtlich hier folgen:

1. Hinterhauptsschuppe des Menschen und des Affen zeigen nach der Geburt nahestehende Gestaltungsverbältnisse.

2. Der Mensch dehnt dieselbe nun nach allen Dimensionen gleichmässig aus.

3. Er erreicht die in sagittaler Ausdehnung absolut unả relativ grösste Hinterhauptsschuppe.

4. Dabei vermehrt sich die Entfernung der Spitze der Hinterhauptsschuppe von der Mitte der Linea semicircularis superior stetig.

5. Die Affen, besonders deren anthropoide Gattungen, vergrössern den oberen Theil der Hinterhauptsschuppe nur in der ersten Jugendzeit und nur in horizontaler Dimension.

6. Die Entfernutíg der Spitze der Hinterhauptsschuppe von der Mitte der Linea semicirc. superior nimmt bei diesen Wesen stetig ab, bei den anthropoiden Gattungen bis zum völligen Verschwinden dieses Raumes, so dass die genannte transversale Linie und die Lambdaränder zu einem Knochenkamme (Crista lambdoidea) verschmelzen.

7. In Einklange damit wird der Winkel, welchen die Lambdaränder an der Spitze der Hinterhauptsschuppe bilden, bei den Affen von der Geburt bis zum vollendeten Wacbstium immer grösser, dem Winkel von 180 Graden zustrebend, während er beim Menschen nie sich vergrössert, sondern dem Verhalten bei der Geburt entweder gleich hleibt oder sich verkleinert.

8. Während die neue Linie, Linea semicircularis suprema, zwischen Spitze und Linea semicirc. superior gelegen, als obere Ansatzgrenze des Musculus cacullaris beim Menschen sich zeitlebens erhält, bleibt für ihr Bestehen bei den Affen kein Raum und geht sie in der Aufwulstung der Lambdaränder unter.

Ich füge binzu:

Die beim Menschen und jugendlichen Affen von der hintern Scheitelgrenze bis zur obern Nackenlinie reichende Hinterhauptsgegend, welche auch die niedersten Menschenracen zeitlebens besitzen, zeigt sich beim erwachseren Affen, besonders bei deren anthropomorphen Arten, auf 0 reducirt. Die obere Nackengrenze ist mit der hintern Scheitelgrenze verschmolzen, und die Nackenmusculatur bis zum Scheitel hinaufgerückt. Gerade der erwachsene anthropoide Affe besitzt in eclatantester Weise keine Hinterhauptsgegend. Den Mangel an Verständniss für den Unterschied in den Tendenzen unserer Arbeiten documentirt Herr Prof. M. auch durch die Worte: „Die Vergleichung des menschlichen Os occipitale mit dem der Affen 
führt J. weiter aus, als ich es für nöthig hielt, und muss ich mich aus Manget ejgner Erfahrung eines Urtheils über diesen Theil der Joseph'schen Untersuchung enthalten " und in den darauf folgenden Passus. - - -

Dass ich zu, den Schlussresultaten des H. Prof. M. entgegengesetzten Ergebnissen komme, liegt nicht, wie Herr Prof. M. irrthümlich meint, in der flüchtigen Benutzung seiner, mir zur Zeit unbekannten, Schrift, sondern, wie leicht ersichtlich, in der Darstellung des Sachverhalts nach meiner Auffassung. Selbst aufmerksamste Lectüre desjenigen Theils seines Schriftchens, welches über Anheftung der Weichtheile handelt, hätte mich nicht, wie Herr Prof. M. meint, vor Fehlern schützen können, wenn meine Ergebnisse fehlerhaft sein sollten, und nicht vermocht, mich zu andern Schussfolgerungen zu drängen.

In Bezug auf den letzten Theil der "Bemerkungen, “ welcher mit den Worten „zum Schlusse" beginnt, dürfte wohl jeder unbefangene Fachgenosse mit mir ausrufen: tant de bruit pour une omelette! Ich wünschte, dass der von Herrn Prof. M. incriminirte Druckfehler, der grösste in meiner Arbeit wäre! Die in der dort gebrauchten Aeusserung: „so hälte es eines Druckfehlers nicht bedurft" liegende Verdächtigung dürfte wohl $H$. Prof. M. zurücknehmen, wenn ich versichere - was aber auch aus Seite 8 meiner Schrift genügend hervorgeht - dass es mir nicht in den Sinn gekommen ist, an der selbständigen Entdeckung des Herrn Prof. M., welchem überdies die Priorität der Veröffentlichung zukommt, irgend wie zu zweifeln.

Nach Vorstebendem muss ich jedem unbefangenen Leser überlassen, zu entscheiden, wer "Denkfebler" gemacht hat, Herr Prof. M., oder ich.

3.

\section{Trichinen in Russland.}

\section{Von Dr. Knoch in St. Petersburg.}

Eine Familie in Petersburg, bestehend aus sieben Personen, erkrankte nach dem Genusse von Schinken und Braunschweiger Wurst gleichzeitig unter Symptomen, welche der zu Rath gezogene Arzt Dr. Knoch als von der Trichinose herrïhrend zu erkenuen glaubte. Da die Untersuchung der Speiseüberreste kein bestimmtes Resultat ergab, stellte derselbe an das Haupt der Familie das Ansuchen, auf operativem Wege den Nacbweis liefern zu dürfen, worauf dieses bereitwillig einging. Bei der am Arme des Patienten in Gegenwart mehrerer anderer Aerzte vorgenommenen Operation erwies sich denn auch sofort das Vorhandensein Jebender Trichinen in der Musculatur, welche, bereits verkapselt, unter dem Mikroskope deutlich erkennbar waren. Die Infection war eine bedeutende, wenn auch die Fälle nicht zu den sehr schweren gehörten. - Es muss bemerkt werden, dass nur noch Reste des Schinkens zur Untersuchung gelangten, da die zu Braunscbweiger (Mett-) Wurst verarbeiteten Theile schon ganz verzehrt waren. 